\title{
BIOPSY OF NONPALPABLE CERVICAL LYMPH NODES: SELECTION CRITERIA FOR ULTRASOUND-GUIDED BIOPSY IN PATIENTS WITH HEAD AND NECK SQUAMOUS CELL CARCINOMA
}

\author{
René H. Kruyt, ${ }^{\dagger}$ Wim L. J. van Putten, ${ }^{+}$Peter C. Levendag, * \\ MAarten F. DE BOER ${ }^{\S}$ and MATthiJs OUdKerK ${ }^{\dagger}$ \\ Departments of ${ }^{\dagger}$ Radiodiagnostics, "Medical Statistics, *Radiation Oncology Head and Neck Surgery, \\ Dr. Daniel den Hoed Cancer Centre, University Hospital Rotterdam, Rotlerdan, The Netherlands
}

(Received 1 August 1995; in final form 11 January 1996)

\begin{abstract}
This article is designed to find an appropriate policy to select nonpalpable cervical lymph nodes in head and neck squamous cell carcinoma patients for ultrasound-guided aspiration biopsy (USB). According to the literature, generally used selection criteria are width of nodes $>10 \mathrm{~mm}$, length-to-width ratio $>2$ and absent echo-rich hilum. In 562 nonpalpable nodes of 355 patients (mean age $60 \mathrm{y}$, range 20-92 y) with head and neck squamous cell carcinoma, a USB procedure was carried out. Nodes were classified according to dimensions and to echo pattern. Representative cytology was obtained in 489 nodes; 112 were classified as malignant. Of the 412 nodes with a width $\leq 10 \mathrm{~mm}, 79$ were malignant. Width is the strongest predictor for malignancy and, if corrected for width, the length-to-width ratio is of no influence. Of the 142 nodes with an echo-poor centre, or an inhomogeneous pattern, $46 \%$ were malignant, compared to $13 \%$ of 342 nodes with an echo-rich centre. It is concluded that selection of lymph nodes of the neck of patients with squamous cell carcinoma of the head and neck should be based on width and echo pattern. We advise subjecting nodes with an echo-rich centre or homogeneous pattern and a width $\geq 4 \mathrm{~mm}$ to USB, and also subjecting nodes with an echo-poor centre or inhomogeneous pattern with a width $\geq 3 \mathrm{~mm}$ to USB.
\end{abstract}

Key Words: Ultrasound, Biopsy, Lymph nodes, Metastases.

\section{INTRODUCTION}

The value of detecting metastatic lymph nodes in the neck of patients with a head and neck neoplasm is twofold. First, detection of metastatic disease in the neck is important with regard to treatment, be it surgery, radiotherapy, chemotherapy or a combination. Second, to estimate the prognosis, if a patient with a squamous cell carcinoma at one side of the head or neck has ipsilateral lymph node metastases the chance of cure may be reduced by $50 \%$ (Batsakis 1979; Marchant et al. 1993; Snow et al. 1982; Spiro et al. 1974). The presence of contralateral metastases leads to a further $50 \%$ reduction in cure rate (Batsakis 1979; Marchant et al. 1993; Snow et al. 1982; Spiro et al. 1974). In case of squamous cell carcinoma of the tongue base, lymph node metastases in patients with no

Address correspondence to: René H. Kruyt, Department of Radiology, Dr. Daniel den Hoed Cancer Centre, University Hospital Rotterdam, Groene Hilledijk 103, 3075 EA Rotterdam, The Netherlands. clinical evidence of lymph node metastases, specimen dissection revealed ipsilateral metastases in $44 \%$ and contralaterally in $22 \%$ (Foot et al. 1993). Apparently, nonpalpable lymph node metastases is not a rare phenomenon in these patients.

Because some $12 \%$ of all tumour-positive neck dissection specimens harbour only micrometastases, one should realize that it will be almost impossible to reach a sensitivity close to $88 \%$ with any prcopcrative staging technique because it is unlikely that micrometastases will be detected by a such a method (Batenburg and Rongen 1990; Feinmesser et al. 1992). In the detection of metastatic lymph nodes in the neck, palpation is characterized by a high false-negative rate of around $25 \%$ and a high false-positive rate between $10 \%$ and $15 \%$ (Feinmesser et al. 1987; Manfredi and Jacobelli 1975; Marchant et al. 1993; Martis et al. 1979; Sako et al. 1964; Shah and Tollefsen 1974). Although ultrasound (US), computed tomography (CT) and magnetic resonance imaging (MRI) are clearly superior to palpation in the detection 
of enlarged lymph nodes in the neck. A disadvantage of these techniques has been difficulty in differentiation between benign and malignant nodes (Gerrits et al. 1994; Johnson 1990; Lenz 1990; Steinkamp et al. 1992, 1994). Other drawbacks of MRI are the high cost, limited availability and practical impossibility to obtain material for pathologic analysis. Drawbacks of CT are the need to administer intravenous contrast media and, compared with US, obtaining material for pathologic analysis is more difficult. Because of the partial volume effect, it is unlikely that metastases with a diameter $<3 \mathrm{~mm}$ will be detected with confidence on CT or MR imaging (Anzai et al. 1994). Ultrasound-guided aspiration biopsy (USB) is characterized by low cost with at least the same accuracy in the detection of lymph nodes in the ncck, and the spatial resolution of US in the area under consideration exceeds that of the other imaging techniques. A problem for all imaging techniques is the differentiation between benign and malignant nodes.

The purpose of this study is to find appropriate selection criteria to indicate USB of lymph nodes in the neck of patients with squamous cell carcinoma of the head and neck area. A number of studies suggest the need for USB for metastases having widths $>10 \mathrm{~mm}$ (Anzai et al. 1994; van den Brekel et al. 1994; Chang et al. 1994; Gerrits et al. 1994; Bruneton et al. 1984, 1986; McLaughlin et al. 1995); others suggest that nodes with widths $>8 \mathrm{~mm}$ should be subjected to USB (Sakai et al. 1988). Some investigators advise USB for nodes $>5 \mathrm{~mm}$ (Hajec 1986; Vassallo et al. 1992). Other published puncture criteria include length-to-width ratio $>2$ and absent echorich hilum (Chang et al. 1994; Sakai et al. 1988, Vassallo et al. 1992).

\section{MATERIALS AND METHODS}

\section{Data and procedures}

From July 1992 to May 1995, a USB procedure was carried out of 562 nonpalpable nodes. This was done in 355 patients (age between 20 and $90 \mathrm{y}$, mean age $60 \mathrm{y}$ ), undergoing 395 US examinations. None of the patients had evidence of nodal neck metastases on clinical examination. Histology of the primary disease was always squamous cell carcinoma.

Image quality during the USB procedures was always such that the needle tip was visible in the node. Vacuum was only applied with the needle tip visible in the node, avoiding aspiration of nonlymphoid material. With this technique, it was possible to obtain adequate material even of nodes behind the jugular vein, with puncture through this vein.

In 156 US examinations, only one node was detected that was subjected to USB. In the remaining 239 examinations two or more nodes were detected.
In 181 examinations with multiple nodes, one node was selected and subjected to USB; in 150 examinations, two nodes were selected for USB; and, in 8 examinations, three or four nodes were subjected to USB. If bilateral nodes were detected, USB was performed on both sides. In case of multiple unilateral nodes, the most caudal node was punctured and if this node had an echo-rich hilum and/or small width, socalled benign characteristics (Chang et al. 1994; Vassallo et al. 1992), the most suspect node at that side was additionally subjected to USB.

Cytologic analyses classified three categories: $1-$ malignant; 2 -benign; and 3-not evaluable. Of the nodes subjected to USB, the size, the echo pattern and the result of cytologic analyses were scored. The size of a node was characterized by its length, defined as the largest diameter on US in millimeters, and width was defined as the maximal diameter perpendicular to the largest diameter in millimeters. The echo pattern was classified as: 1 -echo-rich centre; 2-homogeneous; 3-echo-poor centre; or 4-inhomogeneous. Nodes with an echo-poor or echo-rich centre were classified as eccentric or central. All diagnostic US examinations and USBs were carried out with an Acuson $128 \mathrm{XP} / 10$ (ART) system, using a 7.5-MHz transducer. Initial imaging of the six levels, according to the Sloan-Kettering Memorial Cancer Center (Shah et al. 1981, 1990), of the neck was performed using acoustic gel. The focus was set on $2 \mathrm{~cm}$, allowing a spatial resolution of approximately $0.2 \mathrm{~mm}$ at a distance under the skin of between $3 \mathrm{~mm}$ and $45 \mathrm{~mm}$. After removing the gel from the transducer and from the neck of the patient, $98 \%$ ethanol was applied to the area of cytologic puncture, and the focus was adjusted according to the position of the node. For this purpose, it is sufficient to wipe the skin at the surface of interest with a small gauze, wetted with ethanol.

Biopsies were obtained with a $20 \mathrm{ml}$ syringe and $23 \mathrm{G}$ needle in a manual vacuum device. Under realtime US guidance, cytologic puncture was performed after cleaning the skin and transducer with $98 \%$ ethanol. Acoustic gel was not applied. The transducer was not covered. Vacuum was only applied if the tip of the needle was visible and located in the node. During suction, the needle was moved through a considerable volume of the nodes under continuous US guidance until enough material was aspirated. Material was instantly smeared on microscope slides. No fixation was used and rouline May-Grünwald-Giemsa staining was performed.

\section{Statistical methods}

The primary aim of the analysis was to determine the relationship between US characteristics of nodes and 
cytologic outcome. The fact that some patients underwent multiple US procedures could be disregarded in the main analyses as it turned out that inclusion or exclusion of the data other than those of the first US procedure did no: affect the outcome (details not shown). From these two parameters, the area was calculated as the product of length and width. The size of each node was characterized by its length and its width. As a measure for spherical shape, the ratio of length and width was calculated. A comparison was made of the sizes of the nodes with cy.ology and the sizes of the nodes without cytology. This comparison was restricted to the nodes of patients with multiple nodes, of which only a few were punctured for: cytology.

Of the 562 nodes subjected to cytology, $13 \%$ were nct evaluable (NE), and could therefore not be classified as malignane or benign. Logistic regression analysis was applied to determine whether the probability of NE was related to US pattern or the size of the ncde. A total of 489 nodes had evaluable cytology. Pearson's chi-square test, the Mann-Whitney test and logistic regression analysis were applied to determine which factors were associated with malignancy. The following factors were considered: total number of nodes detected by US, length, width, area, shape, US pattern, and the cytologic findings on other nodes of the same patient of the same US procedure if available.

\section{RESULTS}

Thirty-six patients underwent two or more US procedures. For four patients, the second US was done within 1 month, for four patients, between 1 and 3 months after the first US. For the remaining 28 patients, the second US was done more than 3 months after the first. For the analysis of the relation between US characteristics and cytology, data from all US procedures were included. Cytologic outcome versus width ard versus US pattern is presented in Table 1. As described in "Materials and Methods" ("Data and procedures"), the most caudal node (at both sides in case of hilateral nodes) was selected for USB in case of multiple nodes. If this node had a benign appearance, the most suspect node of the remaining nodes was also subjected to USB. Thus, the selection of nodes was not random. This is also evident from comparison of the sizes of the nodes subjected to cytology and the nodes not subjected to cytology of the 179 patients of whom not all nodes were punctured. For USB, selected nodes were, on average, larger (median area $39 \mathrm{~mm}^{2}$ ys. $28 \mathrm{~mm}^{2}, P=0.0003$, Mann-Whitney test) than nodes not subjected to cytology.

Of the 562 nodes, 73 ( $13 \%$ ) could not be classifred as malignant or benign (Table 1). These nodes were classified as not evaluable (NE). The evaluability of a node primarily depended on its size: of the 40 nodes with length $\leq 4 \mathrm{~mm}, 20 \%$ were NE, whereas, of the nodes with length $>10 \mathrm{~mm}, 10 \%$ were NE. The echo pattern did not show an association with NE (Table 1).

A total of 489 nodes had evaluable cytology, 112 of which were classified as malignant. There is no evidence of positive correlation between the total number of nodes per patient on US and the probability of malignancy. In fact, of the patients with a single node on US, $31 \%$ were malignant, versus $20 \%$ of the nodes of patients with multiple nodes at US. One would expect the cytology of different nodes of the same patient to be correlated. This was confirmed by the analysis. In 219 USB procedures, only one node was punctured, $26 \%$ of which were malignant. In the remaining cases, two, exceptionally three or four, nodes were punctured. Thirteen percent of the 212 nodes with only benign sister nodes were malignant versus $45 \%$ of the 58 nodes with a malignant sister node (Table 1 ). The size of the node, measured by length, width or area, is a strong predictor for malignancy. Width turned out to be the strongest predictor, with $2 \%$ malignancy for nodes with width $\leq 2 \mathrm{~mm}$, increasing to over $70 \%$ for nodes with width over $9 \mathrm{~mm}$ (Table 1). Also, the length of a node shows a strong association with malignancy in a univariate analysis, but not as much as the width. Only a small subgroup of very large nodes (length $\geq 20 \mathrm{~mm}$ ) has a very high proportion of malignancy ( $68 \%)$. The shape of the node, measured by the ratio of length to width, also shows a clear association with the probability of malignancy. None of the 24 nodes with a length-io-width ratio over 3 was malignant. Most of these 24 nodes were small having a width less than $3 \mathrm{~mm}$, and a maximum of $5 \mathrm{~mm}$. Note that a spherical shape is not a reliable indicator for malignancy, as less than one third of the nodes with a length-to-width ratio of less than 2 were malignant. Of the almost spherical nodes (i.e., with a ratio $\leq 1.25, n$ $=58$ ), still only $55 \%$ were malignant.

There were also marked differences in incidence of malignancy related to the US pattern. Of the nodes with an echo-rich centre or a homogeneous pattern, only $13 \%$ were malignant, whereas, of the nodes with an echo-poor centre or inhomogeneous pattern, $46 \%$ were malignant (Table 1). The location of the centre (central or eccentric) was not related with the probability of malignancy (details not shown).

The echo pattern and the size are correlated characteristics: the median width of the nodes with echopoor centre or homogeneous pattern was $6 \mathrm{~mm}$, whereas the nodes with echo-rich centre or inhomogeneous pattern were smaller with a median width of 4 
Table 1. Incidence of malignancy in relation to node characteristics.

\begin{tabular}{|c|c|c|c|c|}
\hline & $\begin{array}{l}\text { Malignant } \\
(n \text { and } \%)\end{array}$ & $\begin{array}{c}P \text {-value of } \\
\text { univariate } \\
\text { analyses }^{\mathrm{a}}\end{array}$ & $\begin{array}{l}\text { Multivariate } \\
\text { odds ratio }\end{array}$ & $\begin{array}{c}P \text {-value of } \\
\text { multivariate } \\
\text { analyses }\end{array}$ \\
\hline Total & $48923 \%$ & & & \\
\hline No. of nodes at US & & 0.52 & & $0.83^{\mathrm{b}}$ \\
\hline 1 & $12331 \%$ & & - & \\
\hline 2 & $12919 \%$ & & - & \\
\hline 3 & $8516 \%$ & & - & \\
\hline 4 & $5721 \%$ & & - & \\
\hline 5 & $3327 \%$ & & - & \\
\hline 6 & $6221 \%$ & & - & \\
\hline Length (mm) & & $<0.0001$ & & $0.60^{b}$ \\
\hline $2.0-4.9$ & $323 \%$ & & - & \\
\hline $5.0-6.9$ & $1047 \%$ & & - & \\
\hline $7.0-8.9$ & $12912 \%$ & & - & \\
\hline $9.0-10.9$ & $8327 \%$ & & - & \\
\hline$>10.9$ & $14148 \%$ & & - & \\
\hline Width (mm) & & $<0.0001$ & & $0.0001^{\mathrm{c}}$ \\
\hline$<2.0$ & $58 \quad 2 \%$ & & - & 0.2 \\
\hline $2.0-4.9$ & $199 \quad 9 \%$ & & - & 1.0 \\
\hline $5.0-6.9$ & $11317 \%$ & & - & 1.7 \\
\hline $7.0-8.9$ & $4949 \%$ & & - & 6.2 \\
\hline $9.0-10.9$ & $2471 \%$ & & m. & 17.5 \\
\hline$>10.9$ & $4672 \%$ & & - & 0.9 \\
\hline Length-to-width ratio & & $<0.0001$ & & $0.03^{\mathrm{b}}$ \\
\hline$<1.5$ & $14938 \%$ & & 1 & \\
\hline $1.5-2$ & $18523 \%$ & & 0.78 & \\
\hline $2-3$ & $13110 \%$ & & 0.49 & \\
\hline$>3$ & $240 \%$ & & & \\
\hline US pattern & & $<0.0001$ & & $<0.001^{\mathrm{d}}$ \\
\hline Homogeneous & $17716 \%$ & & 1 & \\
\hline Echo-rich centre & $1659 \%$ & & $0.8 \%$ & \\
\hline Inhomogeneous & $10743 \%$ & & 2.5 & \\
\hline Echo-poor centre & $4055 \%$ & & 2.7 & \\
\hline Cytology of other nodes, if available & & $<0.0001$ & & $<0.001^{\mathrm{b}}$ \\
\hline No cytology & $21926 \%$ & & $\mathrm{t} 1$ & \\
\hline All benign & $21213 \%$ & & 0.36 & \\
\hline$>1$ malignancy & $5845 \%$ & & 2.3 & \\
\hline
\end{tabular}

${ }^{a} P$ values in univariate analyses derived from logistic regression analyses.

${ }^{\mathrm{b}}$ Adjusted for width and echo pattern.

${ }^{c}$ Adjusted for echo pattern.

${ }^{\mathrm{d}}$ Adjusted for width.

$\mathrm{mm}$. Thus, the difference in size may partly explain the difference in incidence of malignancy, associated with the echo pattern. Multiple logistic regression analysis was done to determine which factors contributed most to a discrimination between malignant and benign nodes. In this analysis, the width of nodes over $9 \mathrm{~mm}$ was set at $9 \mathrm{~mm}$, as no further increase of malignancy with increasing width over $9 \mathrm{~mm}$ was observed. The strongest predictors of malignancy were the width of the node $(P<0.0001)$, and the echo pattern, classified as echo-poor centre or homogeneous versus the others $(P<0.001)$. If these two parameters are known, the length-to-width ratio was only of limited additive value $(P=0.03)$, which was primarily due to the absence of malignancy among the nodes with a ratio over 3 . Nodes with a width over $9 \mathrm{~mm}$ have an odds ratio of malignancy greater than 10 compared to the nodes with a width of between 2 and $4 \mathrm{~mm}$, while the odds ratio for more spherical nodes (length-to-width ratio $\leq 2$ ) compared to the rest is only factor 2 . Other parameters such as length and number of nodes at US showed no association with malignancy if the width and echo pattern are known. The cytologic outcome of other nodes of the same patient, subjected to USB in the same US examination, if available, was still a strong predictor, if width and echo pattern are known. Addition of this factor to the model did not alter the size of the effect of width and echo pattern. Figure 1a shows the observed percentages of malignant nodes in seven classes of nodal width, together with the associated 95\% confidence intervals for nodes with an ccho-rich centre or homogeneous pattern and the curve fitted by the logistic model with $95 \%$ confidence region. Figure $1 \mathrm{~b}$ is similar for nodes with an echo-poor centre or 

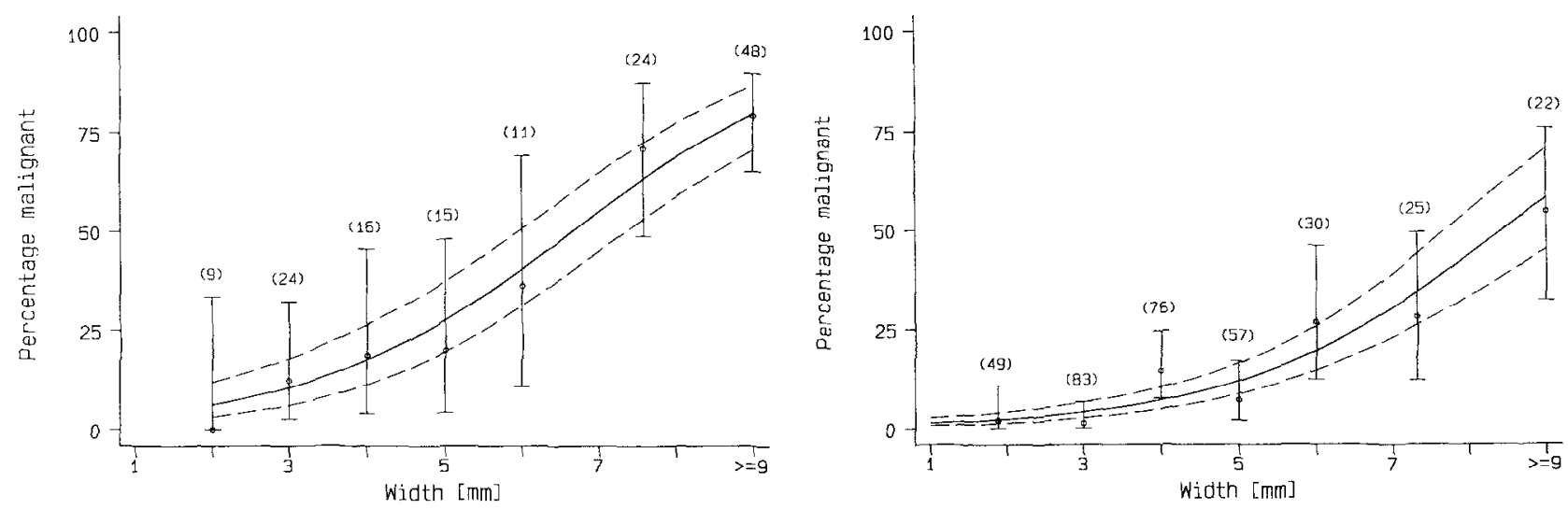

Fig. 1. For nodes with an ccho-rich centre or homogeneous pattern, the observed percentages of malignant nodes are presented in seven classes of nodal width, together with the associated $95 \%$ confidence intervals and the curve fitted by the logistic model with a $95 \%$ confidence region. (b) For nodes with an echo-poor centre or inhomogeneous pattern, the observed percentages of malignant nodes are presented in seven classes of nodal width together with the associated $95 \%$ confidence intervals and the curve fitted by the logistic model with $95 \%$ confidence region. The slope of the fitted curves for nodes with an echo-rich centre or homogeneous pattern (a) is equal to the slope of this fitted curve for nodes with an echo-poor centre or inhomogeneous pattern (b).

in homogeneous pattern. The slopes of the fitted curves are equal. A test on difference of slopes was not statistically significant $(P>0.10)$. In both groups, one observes a clear increase of incidence of malignancy with increasing width. If we consider $10 \%$ as the lower limit for the a priori probability for malignancy based on echo pattern and width, above which USB is indicated, it is useful to calculate from the model in both echo pattern subgroups the width for which the a priori probability of malignancy is equal to $10 \%$. For nodes with an echo-poor centre or inhomogeneous pattern, this width was $2.9 \mathrm{~mm}$ (95\% confidence interval 1.2 $4.5 \mathrm{~mm}$ ), and for nodes with an echo-rich centre or homogeneous pattern it was $4.7 \mathrm{~mm}$ ( $95 \%$ confidence interval $3.0-6.3 \mathrm{~mm}$ ).

\section{DISCUSSION}

\section{The US imaging and USB procedure}

US imaging of the neck is limited to the six levels described by the Sloan-Kettering Memorial Cancer Center (Shah et al 1981; Shah 1990). Althongh medial retropharyngeal nodes will be missed by US imaging, these nodes are of minor or no importance (McLaughlin et al. 1995). Lateral retropharyngeal nodes are important in the prediction of prognosis of the patients under consideration (McLaughlin et al. 1995 ). These nodes lie just medial of the carotid arteries. The majority of these nodes are accessible for US and USB. Only the nodes close to the skull base are inaccessible and will be missed.

If compared with acoustic gel, the use of ethanol as acoustic window induces the limitation of a small volume accessible for imaging. Therefore, cach US examination starts with acoustic gel. The US image quality is not deteriorated by ethanol and the smaller volume to be visualized is of no limitation for USB. If the gel is not removed the cytologic result may be spoiled.

For some patients the USB procedure was painful for several minutes and, in a few patients, the pain did not subside for several days. Because of the morbidity of the procedure, and for practical reasons, not all detected nodes are subjected to USB. Besides pain, no other complications like infections or haemorrhagic problems were observed in this series.

If the a priori probability of malignancy of nodes under consideration is $<10 \%$, one could decide to dispense of USB, but, if this probability is $>10 \%$, USB is advisable. To our knowledge, cut-off percentages for this a priori probability are not reported.

In neck dissection specimens, $74 \%$ of clinically tumour-positive neck sides there is necrosis (Bruneton et al. 1986) with a greater chance of cytologic classification NE. In patients without clinical evidence of lymph node metastases in the neck, however, this percentage is much lower (van den Brekel et al. 1994), yielding a higher rate of positive diagnosis of metastatic disease of these small malignant nodes on cytology. This explains the low proportion of NE nodes in this series. Aspiration was only done with the needle tip under real-time US vision. This is crucial, especially in cases of USB through vascular structures, otherwise too much blood prevents reliable cytologic analyses. No correlation between NE cytology and size 
of the node or echo pattern was found. In all categories of width, NE cytology occurred in around $11 \%$ of cases.

\section{Dimension characteristics}

The most important indication for US and USB is staging of patients with head and neck cancer. If the ultimate goal is to the increase cure rate of the patients under consideration, the group of small malignant nodes is of special interest, for almost all of these nodes are nonpalpable and practically inaccessible for pathologic diagnosis by other techniques such as CT and MRI.

Four size criteria were analyzed: width, length, area and length-to-width ratio. The question is whether the correlation between malignancy and each of the four size and shape parameters may in fact be summarized by one of them. Therefore, multiple logistic regression analysis was performed. Width was the strongest predictor for malignancy. If the width and echo pattern are known, no predictive value was added by the other factors. For the imaging techniques CT, MR and US, it is widely accepted to consider only nodes with a width $>10 \mathrm{~mm}$ as potentially malignant. Only some investigators report a width criterion of $\geq 8$ $\mathrm{mm}$, or $\geq 5 \mathrm{~mm}$. Our findings justify the policy to perform USB in smaller nodes. This difference is due to the smaller nodes subjected to USB in our series. USB was performed from 1-mm width, others do not puncture under 5-mm width. Based on our findings, we advise USB for nodes $\geq 4 \mathrm{~mm}$ wide; however, if the node has an inhomogeneous pattern or echo-poor centre all nodes with a width $\geq 3 \mathrm{~mm}$ should be subjected to USB.

According to several investigators, the length-towidth ratio is of major predictive value. It is stated that the probability of malignancy is $97 \%$ if this ratio is smaller than 2 (Steinkamp et al. 1994). These measurements were made on reconstructed CT images and only nodes with a width $>8 \mathrm{~mm}$ were included in that study. In our series, $68 \%$ of all nodes had a length-towidth ratio smaller than 2 , whereas among the larger nodes ( $\geq 8 \mathrm{~mm}$ ) $92 \%$ had a length-to-width ratio below 2. We also found that the length-to-width ratio is of predictive value. However, the width itself seemed to be a better indicator and, if the width of a node was known, the additional predictive value offered by the length-to-width ratio was only borderline with a $P$ value of 0.03 .

\section{US pattern characteristics}

Nodes with an echo-rich centre are expected to be benign (Hajec 1986; Marchal ct al. 1985; Rubaltelli et al. 1990; Sakai et al. 1988; Vassallo et al. 1992).
In case of thyroid cancer, it is stated that an echorich centre of cervical lymph nodes is such a strong predictor of benignity that USB is not indicated (Hajec 1986). Although the presence of an echo-rich centre in our patients is also positively associated with a lower incidence of malignancy ( $13 \%$ ), the probability of malignancy increases with size and the presence of an echo-rich centre alone is sufficient reason to refrain from USB of these nodes. The echo-rich structure has been attributed to an abundance of lymphocytes in the hilum (Hajec 1986; Sakai et al. 1988) and to fluidfilled sinuses each acting as an acoustic interface (Hajec 1986; Vassallo et al. 1992). If nodes are infiltrated by malignant cells, an eccentric cortical widening is expected to occur more frequently, while in reactive (benign) nodes the entire cortex reacts with global widening as a result (Vassallo et al. 1992). Therefore, we categorized these nodes separately to verify the aforementioned hypothesis. Our data do not support this hypothesis: in our experience, the probability of malignancy for a node with a echo-rich centre in central position is not different from a node with a echorich centre in eccentric position. Therefore, we also advocate performing USB procedures on nodes with an echo-rich centre in the central position if the width is $\geq 4 \mathrm{~mm}$. The analysis of nodes with an homogeneous aspect on US versus the nodes with an echo-rich centre is statistically equivalent. Therefore, we also advocate performing USB procedures on these nodes if the width is $\geq 4 \mathrm{~mm}$.

If a node contains an echo-poor centre, or has an inhomogencous pattern, this is considered to reflect necrosis frequently seen in metastatic nodes. This group of nodes is characterized by a clearly higher percentage of malignancy, but does not exceed $90 \%$ in any category. The need for USB in these nodes is therefore also evident. We subdivided this group of nodes in nodes with: (1) an echo-poor area in the middle; (2) an eccentric echo-poor area; and (3) multiple echo-poor areas (inhomogeneous pattern). There were no substantial differences between these three groups in terms of chance of positive diagnoses of malignancy or representative cytologic material. If the width of these nodes is $\geq 3 \mathrm{~mm}$, the chance to diagnose metastatic disease on cytology exceeds $10 \%$.

\section{CONCLUSION}

We propose that USB should be performed on nodes of patients with squamous cell carcinoma of the head or neck if the a priori risk of malignancy based on size and echo pattern is more than $10 \%$. This implies that, in case of a single neck node at one side of the neck on US, this node should be subjected to USB 
if the width is $\geq 3 \mathrm{~mm}$ in the case of an echo-poor centre or inhomogeneous pattern; and also if the width is $\geq 4 \mathrm{~mm}$ in the case of an echo-rich centre or homogeneous pattern. In case of multiple nodes at one side, the USB should be performed on the node with the largest width and, if possible, with an echo-poor centre or inhomogeneous pattern.

\section{REFERENCES}

Anzai Y, Blackwell KE, Hirschowitz SL. Initial clinical experience with dextran coated supermagnetic iron dioxide for detection of lymph node metastases in patients with head and neck cancer. Radiology 1994; 192:709-715.

Batenburg RJ, Rongen RJ. Ultrasound examination of the head and neck. Thesis. Rotterdam, University Press, 1990.

Batsakis JG. Tumors of the head and neck: Clinical and pathological considerations. Baltimore, MD: Williams and Wilkins, 1979: 144-167, 240-250.

van den Brekel MWM, Castelijns JA, Snow B. Detection of lymph node metastases in the neck: radiologic criteria. Radiology $1994 ; 192: 617-618$.

Bruneton JN, Roux P, Caramella E, Demard F, Vallicioni J, Chauvel P. Ear, nose, and throat cancer: Ultrasound diagnosis of metastasis to cervical lymph nodes. Radiology 1984;152:771-773.

Bruneton JN, Caramella E, Héry M, Aubanel D, Manzino JJ, Picard JL. Axillary lymph node metastases in breast cancer: Preoperative detection with US. Radiology 1986;158:325-326.

Chang DB, Yuan A, Yu CJ, Luh KT, Kuo SH, Yang PC. Differentiation of benign from malignant lymph nodes with colour Doppler sonography. Am J Roentgenol 1994;162:965-968.

Feinmesser R, Freeman JL, Feinmesser M, Noyek AM, Mullen JB. Role of modern imaging in decision making for elective neck dissection. Head Neck 1992; 14:173-176.

Feinmesser R, Freeman JR, Noyek AM, Bril D. Melaslatic neck disease. Arch Otolaryngol Head Neck Surg 1987;113:13071310.

Foote RL, Olsen KD, Davis DL, Buskirk SJ, Stanley RJ, Kunselman SI, Schaid DJ, De Santo LW. Base of tongue carcinoma: Patterns of failure and predictors of recurrence after surgery alone. Head Neck 1993; 15:300-307.

Gerrits CJH, van Overhagen $\mathrm{H}$, van Lom $\mathrm{K}$, Adriaansen HJ, Löwenberg B. Ultrasound examination of pathological cervical lymphnodes in patients with non-Hodgkin's lymphoma and Hodgkin's disease. Br J Haematol 1994;88:626-628.

Hajec PC. Lymph nodes of the neck: Evaluation with IJS. Radiology $1986 ; 158: 739-742$.

Johnson T. A surgeon looks at cervical lymph nodes. Radiology $1990 ; 175: 607-610$.
Lenz M. Computertomographie der Halsweichteile: lymphknoten und ihre Differentialdiagnosen. II Klinische Wertigkeit der CT beim Lymphknotenstaging. Röntgenblätter 1990;43:312-320.

Manfredi D, Jacobelli G. Neck dissection in the treatment of head and neck cancer: Results in 1162 cases. In: Chambers RG, ed. Cancer of the head and neck. Amsterdam: Exerpta Medica, 1975:221-224.

Marchal G, Oyen R, Verschalken J. Sonographic appearance of normal lymph nodes. J Ultrasound Med 1985;4:417-419.

Marchant FE, Lowry LD, Moffitt JJ, Sabbach R. Current trends in posttreatment follow up of patients with squamous cell carcinoma of the head and neck. Am J Otolaryngol 1993; 14:88-93.

Martis C, Karabouta I, Lazaridis N. Incidence of lymph node metastases in elective neck dissection for oral carcinoma. $\mathbf{J}$ Maxillofac Surg 1979; 7:182-191.

McLaughlin MP, Mendenhall WM, Mancuso AA, Parsons JT, et al. Retropharyngeal adenopathy as a predictor of outcome in squamous cell carcinoma of the head and neck. Head Neck $1995 ; 17: 190-198$.

Rubaletti L, Proto E, Salmase R. Sonography of abnormal lymph nodes in vitro: Correlation of sonographic and histologic findings. AJR 1990;155:1241-1244.

Sakai $\mathbf{F}$, Kiyono $\mathrm{K}$, Sone $\mathrm{S}$, et al. Ultrasonic evaluation of cervical metastatic lymphadenopathy. J Ultrasound Med 1988; 7:305310.

Sako K, Pradier RN, Marchetta FC, Pickren RW. Fallability of palpation in the diagnosis of metastases to cervical nodes. Surg Gynecol Obstet 1964;118:989-990.

Shah JP. Cervical lymph node metastases - diagnostic, therapeutic, and prognostic implications. Oncology 1990;4:61-69.

Shah JP, Strong E, Spiro RH, Vikram B. Neck dissection: Current status and future possibilities. Clin Bull 1981;11:25-33.

Shah JP, Tollefsen HR. Epidermoid carcinoma of the supraglottic larynx: Role of neck dissection in initial surgical treatment. Am J Surg 1974: 128:494-499.

Snow GB, Annyas AA, Sloten AE, Bartelink H, Hartelink AAM Prognotic factors of neck node metastases. Clin Otolaryngol 1982; 7:185-192.

Spiro RH, Alfonso AE, Farr HW, Strong EW. Cervical node metastases from epidermoid carcinoma of the oral cavity and oropharinx. A critical assessment of current staging. Am J Surg 1974; 128:562-567.

Steinkamp HJ, Hosten N, Langer R, Mathe F, Ehritt C, Felix R. Halslymphknotenmetastasen: Sonographischer Malignitätsnachweis. Fortschr Röntgenstr 1992;156:135-141.

Steinkamp HJ, Hosten N, Richter C, Schedel H, Felix R. Enlarged cervical lymph nodes at helical CT. Radiology 1994; 191:795798.

Vassallo P, Wernecke K, Roos N, Peters PE. Differentiation between benign from malignant superficial lymphadenopathy: The role of high resolution US. Radiology 1992;183:215-220. 